ANNALES

UNIVERSITATIS MARIAE CURIE-SKŁODOWSKA

LUBLIN - POLONIA

VOL. LXXII, 1

SECTIO AA

2017

\title{
Study of the influence of the binary mixtures of fluorocarbon surfactants on the surface tension of water
}

\author{
Anna Taraba* and Katarzyna Szymczyk \\ Department of Interfacial Phenomena, Faculty of Chemistry, \\ Maria Curie-Skłodowska University, \\ Maria Curie-Skłodowska Sq. 3, 20-031 Lublin, Poland \\ *anna.taraba@poczta.umcs.lublin.pl
}

Influence of the binary mixtures of fluorocarbon surfactants Zonyl FSO-100 (FSO100) and Zonyl FSN-100 (FSN100) on the surface tension of the water was studied. The effectiveness of adsorption process of the surfactant at the water-air interface was calculated from the obtained values of the surface tension of studied mixtures. Also the maximum surface area per molecule of surfactant in the interfacial area $A_{m}$ and the free energy at the water-air interface $\Delta G_{\min }^{S}$ were determined. Moreover, the existence of synergetism or antagonism effect, which reduces the surface tension of the water was examined. On the basis of the Rosen's model parameter the values of intermolecular interactions in the mixed monolayer adsorption were calculated.

\section{INTRODUCTION}

Surface active agents (surfactants) are the substances which reduce the surface tension of the medium in which they are dissolved, and/or the interfacial tension with other phases, and, accordingly, are positively adsorbed at the liquid/vapour and/or other interfaces. The term surfactant is also applied correctly to sparingly soluble substances, which decrease the surface tension of a liquid by spreading spontaneously over its surface. The surfactants can aggregate in their solutions making micelles. 
This two processes adsorption and micellization are fundamental for the surfactants [1].

On the other hand, fluorocarbon amphiphile is characterized by unique properties such as stronger surface activity and formation of micelles as well as other self-assemblies at lower concentrations in comparison with the corresponding hydrogenated analogue $[1,2]$. Perfluorocarbon chains are much more hydrophobic than the corresponding hydrocarbon ones and have a pronounced lipophobicity, which is estimated to be about one-third the free energy of transfer of a methylene group from alkane to water [3]. Furthermore, the low polarizability of fluorine atoms results in very weak van der Waals interactions between perfluorocarbon chains. The combination of fluororacbon and hydrocarbon surfactants generates unusual and interesting phenomena such as miscibility, phase separation, and compartmentalization for micelles, liposomes, and Langmuir monolayers [4-6]. Also mixtures of fluorocarbon surfactants are very important due to their high surface activity and chemical stability. Thus the purpose of the presented studies was to determine the influence of the concentration and composition of binary mixtures of two nonionic fluorocarbon surfactants, Zonyl FSN-100 (FSN100) and Zonyl FSO-100 (FSO100), on the surface tension of water at $293 \mathrm{~K}$. The interactions between surfactants in the surface layers were also investigated and compared to the properties of single surfactants and their mixtures at the water-air interface.

Zonyl FSN-100 (FSN100) and Zonyl FSO-100 (FSO100) are ethoxylated nonionicfluorosurfactants, which are applied as effective additives to organic polymers as well as solvent-based paints and coatings $[7,8]$. Zonyl FSN-100 by weight of press-ready ink can improve cylinder life by $25-40 \%$ depending on the ink and cylinder, and improve the print quality by reducing "snowflaking" and streaking of certain inks and keeping ink viscosity [8]. Zonyl FSN-100 is also used in cleaning formulations which facilitates the removal of calcium sulphate scale from a reverse osmosis membrane [7].
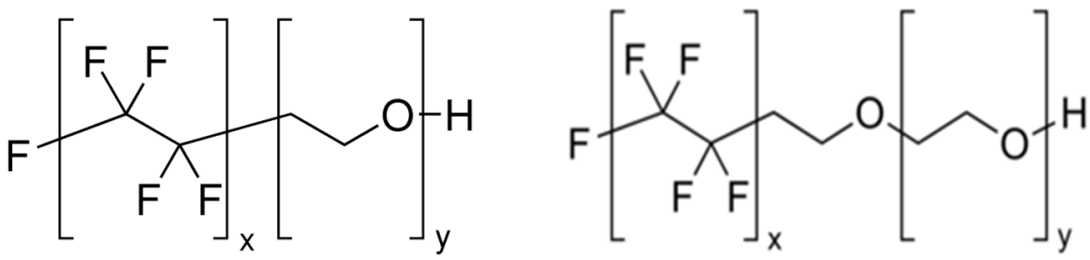

Fig. 1. The structure of Zonyl FSN-100 (on the left, $x=6, y=14$ ) and Zonyl FSO-100 (on the right, $\mathrm{x}=5, \mathrm{y}=10$ ). 


\section{EXPERIMENTAL}

Aqueous solutions of FSN100 and FSO100 mixtures at different molar fractions in the bulk phase, $\alpha$, were prepared using doubly distilled and deionized water (Destamat Bi18E). The surface tension of water was always controlled before the solution preparation.

The following mixtures were prepared:

$$
\begin{aligned}
& \text { FSN100+ FSO100 }(\alpha \text { FSN100 }=0.2)-\mathrm{M}_{\mathrm{a}}, \\
& \text { FSN100+ FSO100 }(\alpha \text { FSN100 }=0.4)-\mathrm{M}_{\mathrm{b}}, \\
& \text { FSN100+ FSO100 }(\alpha \text { FSN100 }=0.6)-\mathrm{M}_{\mathrm{c}}, \\
& \text { FSN100+ FSO100 }(\alpha \text { FSN100 }=0.8)-\mathrm{M}_{\mathrm{d}} .
\end{aligned}
$$

Surface tension measurements were made at $293 \mathrm{~K}$ with a Krüss K9 tensiometer under atmospheric pressure by the ring method. The platinum ring was thoroughly cleaned, and the flame dried before each measurement. In all cases more than 10 successive measurements were carried out, and the standard deviation did not exceed $\pm 0.2 \mathrm{mN} / \mathrm{m}$ temperature was controlled within $\pm 0.1 \mathrm{~K}$.

\section{RESULTS AND DISCUSSION}

From the values of the surface tension of studied binary mixtures of FSN100 and FSO100 (Fig. 1) as well as the literature data of the surface tension of single surfactants [9], it results that at a concentration in the bulk phase equal to $10^{-6} \mathrm{M}$ mixture $\mathrm{M}_{\mathrm{c}}$ reveals the greatest reduction of the surface tension of water $\left(\gamma_{L V}=57.55 \mathrm{mN} / \mathrm{m}\right)$ while single surfactant FSO100 the lowest $\left(\gamma_{L V}=66.7 \mathrm{mN} / \mathrm{m}\right)$ (Fig. 2). At the concentration equal to $10^{-4} \mathrm{M}$, that is higher than the values of the critical micelle concentration of the studied mixtures (Table 1), it is quite different that is the smallest value of $\gamma_{L V}(21.6 \mathrm{mN} / \mathrm{m})$ is observed for the binary mixture of surfactants at the molar fraction $\alpha$ FSN100 $=0.2\left(\mathrm{M}_{\mathrm{a}}\right)$, and the greatest one for FSN100 $\left(\gamma_{L V}=28.5 \mathrm{mN} / \mathrm{m}\right)$. These differences in $\gamma_{L V}$ values at the same concentrations indicates that the efficiency of the adsorption process at the air-water interface of studied solutions is different.

A convenient measure of the adsorption process efficiency is the concentration of surfactant, which is required to reduce the surface tension of water by $20 \mathrm{mN} / \mathrm{m}, C_{20}$. The calculated values of $C_{20}$ para- 
meter of the studied mixtures and those taken from the literature $[9,10,11,12]$ (Table 1) are arranged in the following order:

$$
\mathrm{M}_{\mathrm{c}}<\mathrm{M}_{\mathrm{b}}<\mathrm{FSN} 100<\mathrm{M}_{\mathrm{a}}<\mathrm{M}_{\mathrm{d}}<\mathrm{FSO} 100
$$

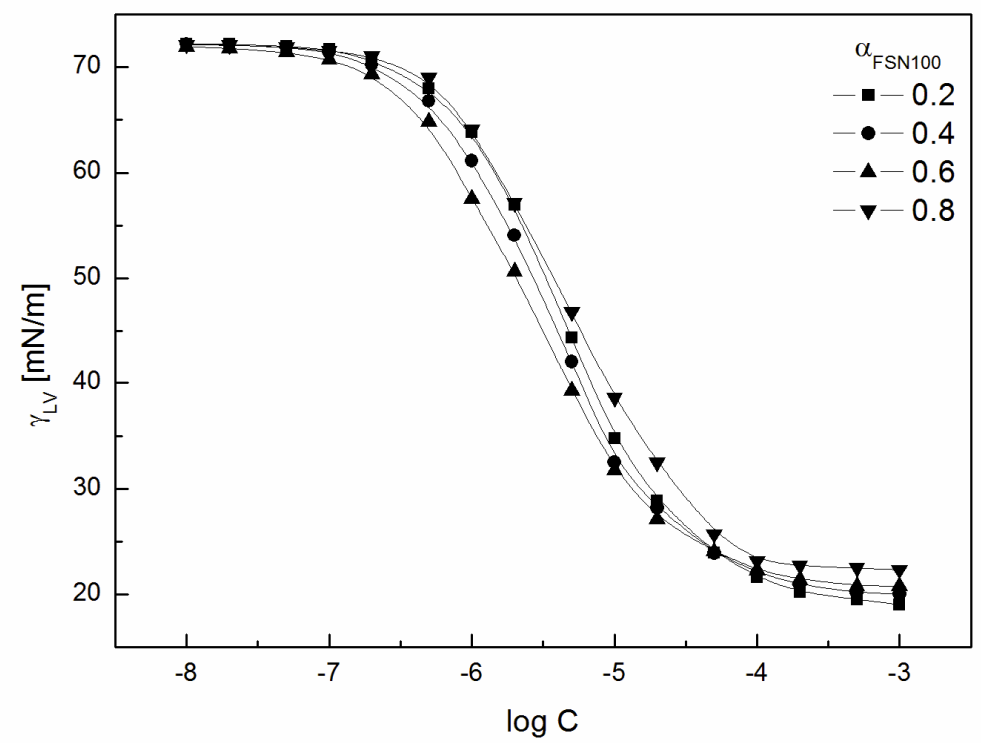

Fig. 2. The relationship of the values of $\gamma_{L V}$ of the binary mixtures of fluorocarbon surfactants FSO100 and FSN100 versus $\log C$

Table 1. Values of the calculated parameters $C M C, C_{20}, \Gamma_{m}, A_{m}, \Delta G_{\text {min }}^{S}$ for the mixtures under investigations.

\begin{tabular}{ccccc}
\hline & $\mathrm{M}_{\mathrm{a}}$ & $\mathrm{M}_{\mathrm{b}}$ & $\mathrm{M}_{\mathrm{c}}$ & $\mathrm{M}_{\mathrm{d}}$ \\
\hline$C M C\left[\mathrm{~mol} / \mathrm{dm}^{3}\right]$ & $2.34 \cdot 10^{-5}$ & $2.16 \cdot 10^{-5}$ & $2.24 \cdot 10^{-4}$ & $3.61 \cdot 10^{-5}$ \\
$C_{20}$ & $2.71 \cdot 10^{-6}$ & $2.15 \cdot 10^{-6}$ & $1.61 \cdot 10^{-6}$ & $2.94 \cdot 10^{-6}$ \\
$\Gamma_{m}\left[\mathrm{~mol} / \mathrm{m}^{2}\right]$ & $5.66 \cdot 10^{-6}$ & $5.48 \cdot 10^{-6}$ & $4.82 \cdot 10^{-6}$ & $4.72 \cdot 10^{-6}$ \\
$A_{m}\left[\mathrm{~nm}^{2}\right]$ & 0.293 & 0.303 & 0.344 & 0.352 \\
$\Delta G_{\text {min }}^{S}$ & 3.919 & 3.642 & 4.337 & 4.743 \\
\hline
\end{tabular}

As follows the mixture $\mathrm{M}_{\mathrm{c}}(\mathrm{FSN} 100+\mathrm{FSO} 100, \alpha$ FSN100 $=0.6)$ shows the greatest efficiency in the reduction of the surface tension of water, as the value $C_{20}$ is achieved already at a concentration equal to 
$1.61 \cdot 10^{-6} \mathrm{M}$ and the lowest efficiency is exhibited by FSO100 $\left(C_{20}=4.74 \cdot 10^{-6} \mathrm{M}\right)[9,10]$.

Taking into account the obtained values of the surface tension of studied mixtures, it is also possible to determine the effectiveness of adsorption process of the surfactant at the water-air interface on the basis of the values of the surface excess concentration at the surface saturation, $\Gamma_{m}$, surface excess concentration of surfactants at the water-air interface can be determined on the basis of the adsorption isotherms using the Gibbs equation $[5,9,12,13]$.

For the dilute solution $\left(10^{-2} \mathrm{~mol} / \mathrm{dm}^{3}\right.$ or less $)$ containing a nonionic surfactant, the Gibbs equation can be written in the form:

$$
\Gamma=-\frac{1}{R T} \frac{d \gamma_{L V}}{d \ln C}
$$

where $C$ is the concentration of surfactant and $\gamma_{L V}$ its surface tension. The concentration of each surfactant mixture at the interface at the surface saturation can be calculated from the slope of $\gamma_{L V}-\ln C$ plot.

Assuming the monolayer adsorption it can be stated that the amount of adsorbed substance is inversely proportional to the surface area per molecule of surfactant in the interfacial area, which may be expressed by the equation:

$$
A=\frac{1000}{6.023 \Gamma}
$$

where $\Gamma$ is expressed in $\mu \mathrm{mol} / \mathrm{m}^{2}$.

Table 1 shows that the values $\Gamma_{m}$ are arranged in the following order:

$$
\text { FSN100 }<\text { FSO100 }<\mathrm{M}_{\mathrm{d}}<\mathrm{M}_{\mathrm{c}}<\mathrm{M}_{\mathrm{b}}<\mathrm{M}_{\mathrm{a}}
$$

From the comparison of the values of $\Gamma_{m}$ (Table 1), it results that the binary mixture $\mathrm{M}_{\mathrm{a}}$ exhibits the highest effectiveness in reducing the surface tension of water, because it has the highest $\Gamma_{m}$ value thus the value of $A_{m}$ is the smallest. Knowing the values of $A_{m}$ the free energy, $\Delta G_{\text {min }}^{S}$, at the water-air interface can be determined. $\Delta G_{\text {min }}^{S}$ is regarded as the work needed to make an interface per mole or the free energy change accompanied by the transition from the bulk phase to the surface phase of the solution components and is defined as $[10,11]$ :

$$
\Delta G_{\min }^{S}=A_{m} \cdot \gamma_{C M C} \cdot N
$$

where $\gamma_{C M C}$ is the surface tension of the solution at the CMC. 
In other words, the lower the value of $\Delta G_{\min }^{S}$, the more thermosdynamically stable surface is formed. It follows from Table 1 that the mixture $\mathrm{M}_{\mathrm{b}}$ formed the surface that is the most thermodynamically stable.

The comparison of the calculated values of $\Delta G_{\min }^{S}$ and $\Gamma_{m}$ for the studied solutions and those for the single surfactants, FSN100 and FSO100 [9, 12] indicates synergism in the surface tension reduction efficiency for binary mixtures.

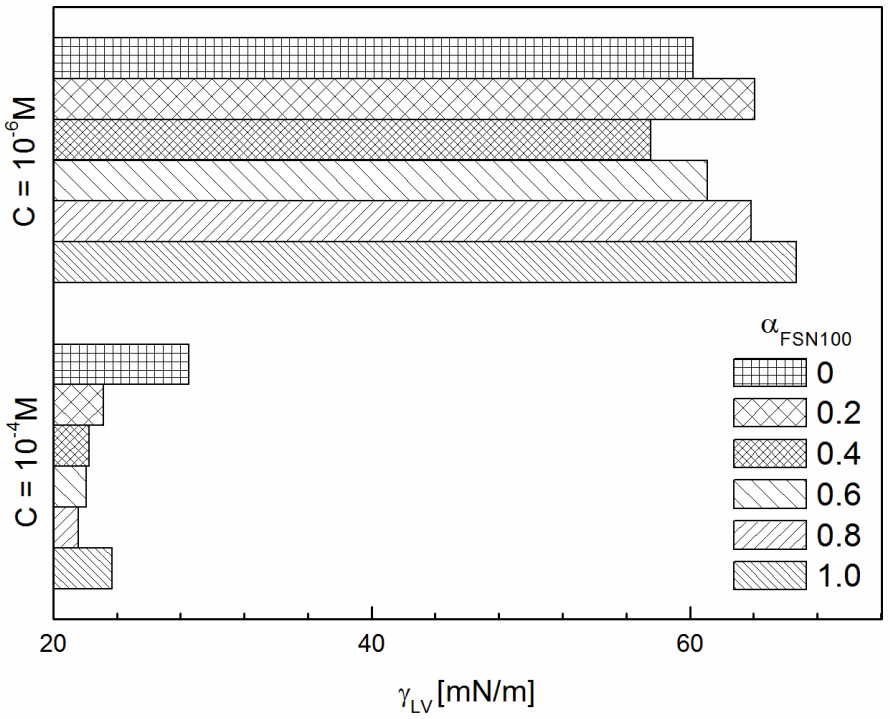

Fig. 3. The relationship of the values of $\gamma_{L V}$ of the binary mixtures of fluorocarbon surfactants FSO100 and FSN100 at their concentrations: $10^{-4}$ and $10^{-6} \mathrm{~mol} / \mathrm{dm}^{3}$.

The first step to establish and explain this effect is to calculate the composition of the mixed monolayer adsorption, and on its basis the parameters of intermolecular interactions. There are many theories, which allow to determine the parameters of intermolecular interactions in the monolayer adsorption consisting of two different surfactants. One of the frequently used is the Rubingh and Rosen theory [1]. This theory introduces the following relationships:

$$
\frac{X_{1}^{2} \ln \left(\alpha C_{12} / X_{1} C_{1}^{0}\right)}{\left(1-X_{1}\right)^{2} \ln \left[(1-\alpha) C_{12} /\left(1-X_{1}\right) C_{2}^{0}\right]}=1
$$




$$
\beta^{\sigma}=\frac{\ln \left(\alpha C_{12} / X_{1} C_{1}^{0}\right)}{\left(1-X_{1}\right)^{2}}
$$

where $\alpha$ is the mole fraction of surfactant 1 in the surface mixture in solution, $X_{1}$ is the mole fraction of surfactant 1 in the mixed monolayer (1- FSN100). The values $C_{1}^{0}, C_{2}^{0}, C_{12}$ are molar concentration of surfactants 1 and 2, and their mixture in solution respectively, which are necessary to reduce the surface tension of the given value; $\beta^{\sigma}$ is a parameter of intermolecular interactions in the mixed monolayer at the solution-air interface, the subscripts 11,22 and 12 refer to interactions between like molecules $(11,22)$ and dissimilar molecules $(12)$.

Using this theory the mole fractions of the surfactant in the mixed adsorption monolayer at the water-air interface were calculated, $X_{1}$ and the intermolecular interaction parameter, $\beta^{\sigma}$ for the examined mixtures with $\gamma_{L V}=60.55,52.8,50,45,40,35$, and $30 \mathrm{mN} / \mathrm{m}$ and they are presented in Fig. 3 and 4. It follows from Fig. 3 that the molar fraction of the fluorocarbon surfactant FSN100 in a mixed adsorption monolayer for all tested mixtures decreases with the decrease of the values of the surface tension $\gamma_{L V}$ of the solution.

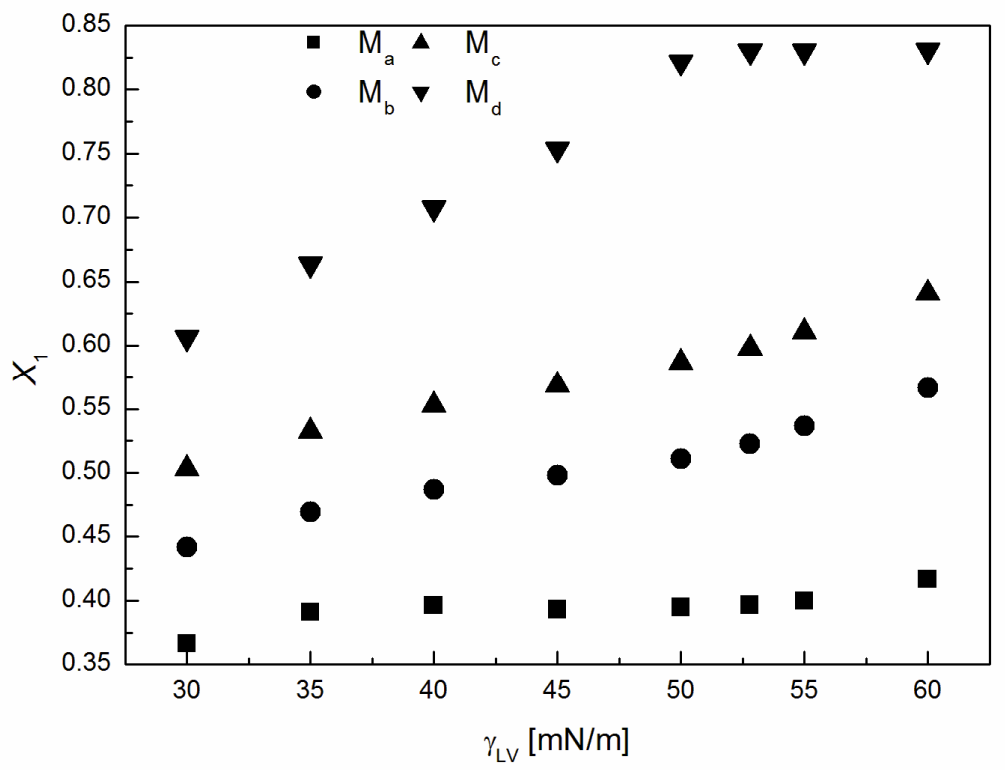

Fig. 4. The relationship of the molar fraction, $X_{1}$, FSN100 in the mixed adsorption monolayer at the water-air interface mixtures formed from the solution, $M_{a}, M_{b}$, $\mathrm{M}_{\mathrm{c}}, \mathrm{M}_{\mathrm{d}}$ versus $\gamma_{L V}$ surface tension of the solution. 


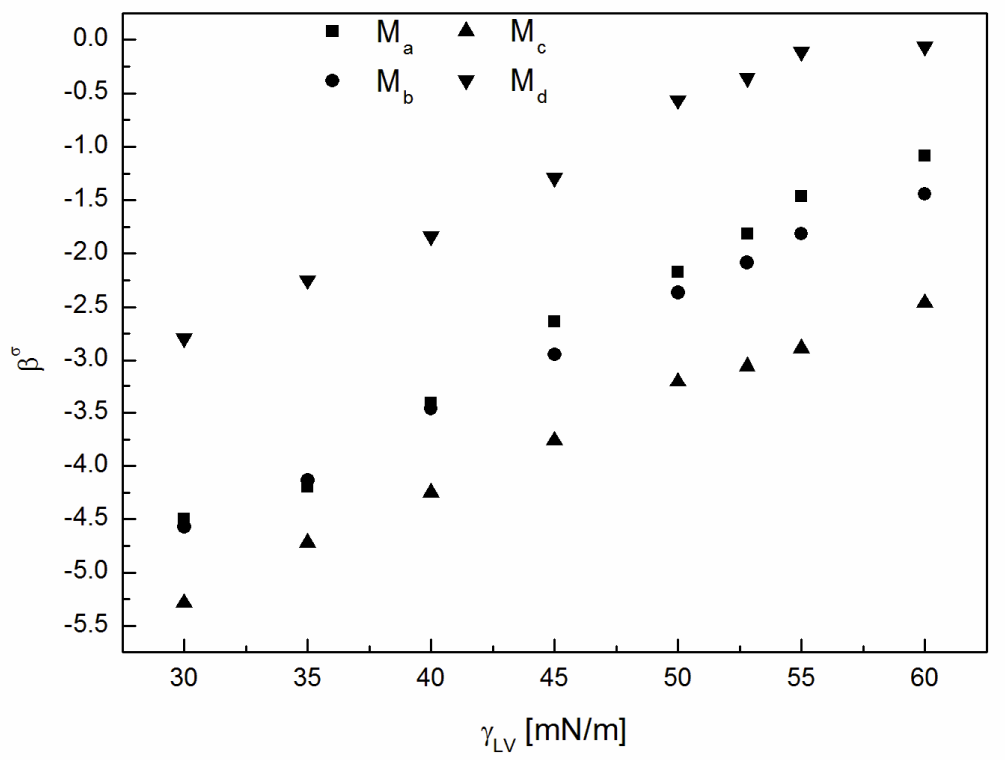

Fig. 5. The relationship of $\beta^{\sigma}$ parameter of the aqueous solutions of the mixtures $M_{a}, M_{b}, M_{c}, M_{d}$ versus $\gamma_{L V}$ surface tension of the solution.

The series of parameters describing efficiency and effectiveness of the adsorption process does not overlap. These differences can be probably explained on the basis of geometric shapes of surfactant particles, size of the hybridized hydrophilic groups and that of electrostatic interactions or other interactions between these groups.

Fig. 4 shows the values of intermolecular interactions in mixed monolayers of adsorption $\beta^{\sigma}$. They are negative and like the values of $X_{1}$ change with the decrease of the mixture surface tension. The negative values of $\beta^{\sigma}$ parameter, the lowest in the case of the mixture $\mathrm{M}_{\mathrm{d}}$, indicate a synergistic effect of the studied mixtures in the reduction of the water surface tension. However, in accordance with the Rubingha and Rosen theory $\beta^{\sigma}$ value is necessary in the presence of synergism or antagonism in reducing the surface tension of water. In the case of a mixture of two surfactants, synergism in the reduction of $\gamma_{L V}$ occurs if the surface tension of an aqueous solution of the surfactant is obtained at the lower mixture concentration that of any of the surfactants and when the $\left|\beta^{\sigma}\right|>\mid \ln \left(C_{1}^{0} /\right.$ $\left.C_{2}^{0}\right) \mid$ is accomplished. $\left|\ln \left(C_{1}^{0} / C_{2}^{0}\right)\right|$ for $60 \mathrm{mN} / \mathrm{m}$ is equal 0.74 ; for $55 \mathrm{mN} / \mathrm{m}$ is $0.69 ; 52,8 \mathrm{mN} / \mathrm{m}=0.59 ; \quad\left|\ln \left(C_{1}^{0} / C_{2}^{0}\right)\right|$ for $45 \mathrm{mN} / \mathrm{m}=0.39$; for $40 \mathrm{mN} / \mathrm{m}$ is equal $0.26 ; 35 \mathrm{mN} / \mathrm{m}=0.03$ and $\left|\ln \left(C_{1}^{0} / C_{2}^{0}\right)\right|$ for $30 \mathrm{mN} / \mathrm{m}$ is 0.36 . 
Comparing the values of $\left|\beta^{\sigma}\right|$ and $\left|\ln \left(C_{1}^{0} / C_{2}^{0}\right)\right|$ it can be stated that all mixtures except from $\mathrm{M}_{\mathrm{c}}$ (at low concentrations), exhibit a synergistic effect in reducing the surface tension of water, $\gamma_{L V}$ throughout the changes of the values of surface tension.

\section{CONCLUSIONS}

The measurements of surface tension of aqueous solutions of binary mixtures of the fluorocarbon surfactants, FSN100 and FSO100, and analysis of their values allow to draw the conclusion that the surface tension of aqueous solutions of the studied mixtures depends on both the composition and total concentration. Also at a concentration at which the surface tension of water is reduced by $20 \mathrm{mN} / \mathrm{m}$, from the tested systems of binary mixture, $\mathrm{M}_{\mathrm{c}}$ has the highest effectivness in reducing the surface tension of water $\left(C_{20}=1.61 \times 10^{-6}\right)$, while FSO100 has the lowest efficiency in the process $\left(C_{20}=3.74 \times 10^{-6}\right)$. The molar fractions of the mixture of surfactants in a mixed adsorption monolayer are determined on the basis of surface tension and Rosen's model for all tested mixtures with the decreasing surface tension. Moreover the parameter values of the intermolecular interactions in the mixed adsorption monolayer calculated from Rosen's model and specific conditions of synergetizm or antagonism occurrence to reduce the surface tension of the water show that a mixture of $\mathrm{M}_{\mathrm{c}}$ throughout values of surface tension shows a synergistic effect in reducing the surface tension of water.

\section{REFERENCES}

[1] J.M. Rosen, Phenomena in Mixed Surfactant Systems, ACS Symposium Series 311, Washington, 2004.

[2] R.M. Hill, Mixed Surfactant Systems, Surfactant Science Series 46, MarcelDekker, NewYork, 1993.

[3] B. Binks, P. Fletcher, S. Kotsev, R. Thompson, Adsorption and Aggregation of Semifluorinated Alkanes in Binary and Ternary Mixtures with Hydrocarbon and Fluorocarbon Solvents, Langmuir, 1997, DOI: $10.1021 / \mathrm{la} 970408$ i.

[4] K. Szymczyk, Properties of the ternary mixtures of fluorocarbon and hydrocarbon nonionic surfactants at the water-air interface, Journal of Fluorine Chemistry, 2013, DOI: 10.1016/j.jfluchem.2013.01.036. 
[5] J.M. Rosen, Molecular interactions and the quantitative prediction of synergism in mixtures of surfactants, Progress in Colloid and Polymer Science, 1998, DOI: 10.1007/BFb0118155.

[6] X.Y. Hua, J.M. Rosen, Calculation of the coefficient in the Gibbs equation for the adsorption of ionic surfactants from aqueous binary mixtures with nonionic surfactants, Journal of Colloid Interface Science, 1982, DOI: 10.1016/0021-9797(82)90344-7.

[7] H. Kunieda, K. Shinoda, Krafft points, critical micelle concentrations, surface tension, and solubilizing power of aqueous solutions of fluorinated surfactants, Journal of Physical Chemistry B, 1976, DOI: 10.1021/ j100563a007.

[8] J.L. Blin, M.J. Stebe, Perfluorodecalin Incorporation in Fluorinated Surfactant-Water System: Tailoring of Mesoporous Materials Pore Size, Journal Physical Chemistry, 2004, DOI: 10.1021/jp049379s.

[9] M.P. Kraft, Fluorocarbons and fluorinated amphiphiles in drug delivery and biomedical research, Advanced Drug Delivery Reviews, 2001, DOI: 10.1016/S0169-409X(01)00107-7.

[10] K. Szymczyk, Wettability of polymeric solids by ternary mixtures composed of hydrocarbon and fluorocarbon nonionic surfactants, Journal of Colloid and Interface Science, 2011, DOI: 10.1016/j.jcis.2011.07.029.

[11] E. Kissa, Fluorinated Surfactants, Synthesis, Properties, Applications, Surfactant Science Series, Marcel Dekker, New York, 1994.

[12] E. Kissa, Fluorinated Surfactants and Repellents, Surfactant Science Series, Marcel Dekker, New York, 2001.

[13] K. Szymczyk, Behaviour of the fluorocarbon surfactants in the monolayer at the water-air interface and in the bulk phase, Journal of Fluorine Chemistry, 2013, DOI: 10.1016/j.jfluchem.2013.02.018.

[14] J. Ościk, Adsorption, Państwowe Wydawnictwo Naukowe, Warsaw, 1979. 